\title{
Thermomechanical FEM-based modelling for semi-crystalline polymers exhibiting the double yield phenomenon
}

\author{
P. Hao ${ }^{\dagger, *}$, V. Laheri ${ }^{\dagger}$ and F.A. Gilabert ${ }^{\dagger}$ \\ ${ }^{\dagger}$ Department of Materials, Textiles and Chemical Engineering \\ Ghent University, Building 46, 9052 Zwijnaarde, Belgium \\ Email: pei.hao@ugent.be, vikram.laheri@ugent.be, fran.gilabert@ugent.be \\ Web page: https://materialmodels.ugent.be \\ * SIM M3 program, 9052 Zwijnaarde, Belgium
}

\begin{abstract}
Double yield is a common phenomenon observed in a variety of semi-crystalline polymers (SCPs). Although abundant constitutive models have been developed targeting SCPs, either in a micromechanics framework [1] or in a homogenous approach with explicit consideration of crystallization degree [2], the time-dependent double yield points still pose difficulties to be quantitively captured. Under quasi-static compressive load, the true stress can experience an increase of $40 \%$ between the first and second yield points [3]. Tests conducted at moderate strain rates reveal a characteristic "hump" at the first yield taking place in the amorphous phase. Increasing the applied strain-rate also rises both yield points, although possible thermal softening due to self-heating might cause a dropdown trend under large strains.
\end{abstract}

We introduce a new approach to tackle SCPs more practically under the finite strain kinematic framework. The first yield point is assumed to take place in the amorphous phase. This phase is described by a physicalbased visco-plastic amorphous model that relies on the double kink theory for glassy polymers [4]. The second yield is captured by using a rate-independent elasto-plastic response providing a simplification of the plastic flow taking place in some metal-based poly-crystalline materials. To connect both responses in a meaningful way, a procedure to operate the inelastic deformation gradient of the crystalline phase via the right stretch tensor is proposed. A characteristic time is introduced to control the saturated state in the amorphous phase and disclose the "hump". The temperature evolution is updated by solving the energy balance equation via plastic dissipation, which is used to feed the temperature-dependent properties. The simulated results show that the proposed thermomechanical constitutive model is capable to efficiently predict the double yield phenomenon as well as the stress-strain curves at different strain rates with minimum experimental datasets.

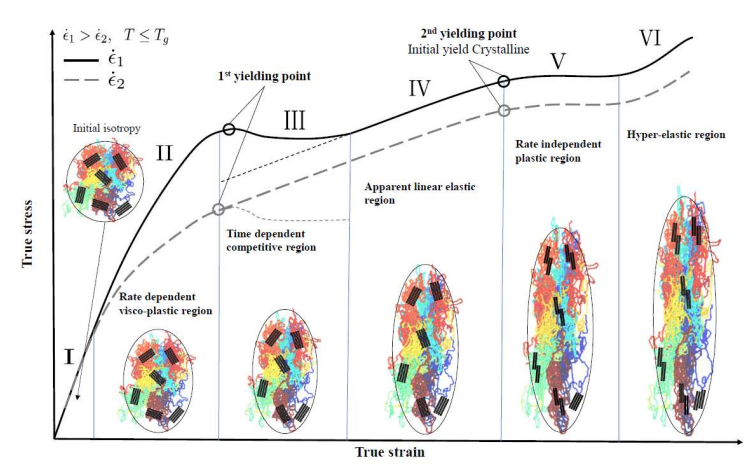

Figure 1. Schematical illustration of mechanical behaviour of semi-crystalline polymers affected by amorphous and crystalline phases.

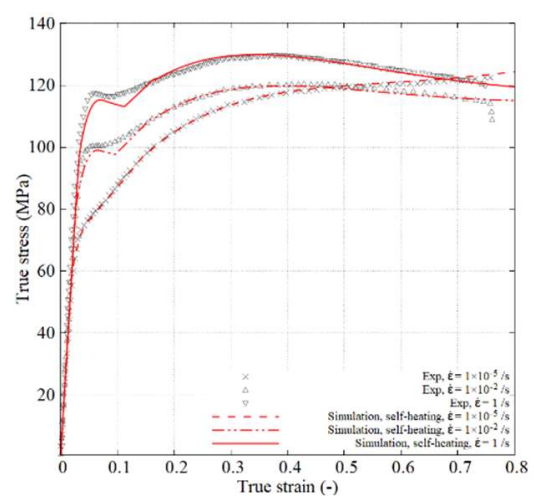

Figure 2. Comparison between simulated results and experimental ones from literature for nylon 101 [3].

\section{REFERENCES}

[1] M. Uchida and N. Tada, "Micro-, meso- to macroscopic modeling of deformation behavior of semi-crystalline polymer," Int. J. Plast., 49, 164-184, (2013).

[2] G. Ayoub et al., "Effects of crystal content on the mechanical behaviour of polyethylene under finite strains: Experiments and constitutive modelling," Int. J. Plast., 27, 492-511, (2011).

[3] A. S. Khan and B. Farrokh, "Thermo-mechanical response of nylon 101 under uniaxial and multi-axial loadings: Part I, Experimental results over wide ranges of temperatures and strain rates," Int. J. Plast., 22, 1506-1529, (2006).

[4] K. A. Chowdhury, A. A. Benzerga, and R. Talreja, "A computational framework for analyzing the dynamic response of glassy polymers," Comput. Methods Appl. Mech. Engrg., 197, 4485-4502, (2008). 